\title{
http://bjas.journals.ekb.eg \\ Endothelial Cell Changes after Implantable Collamer Lens Surgery in Eyes with Different Anterior Chamber Depth \\ S.R.Abbas, T.I.Salm and A.A.Mahmoud
}

Ophthalmology Dept., Faculty of Medicine, Benha Univ., Benha, Egypt

E-Mail:ophthalamology@gmail.com

\begin{abstract}
For a long time, many trials have been done in order to correct refractive errors. This was either with classical approaches such as spectacles and contact lenses or by refractive surgeries which have become an acceptable alternative for a large number of patients and surgeons over the past three decades. Phakic IOL with its different types has become one of surgical alternatives in order to correct refractive errors.. The present work studied the effect of anterior chamber depth on corneal endothelium cell count and morphology including central corneal thickness (CCT), cell density (CD), coefficient of variation (CV) after implantable collamer lens (ICL) surgery. Our study included twenty eyes with high myopia. Ten eyes, with AC depth below $3.50 \mathrm{~mm}$. Other twenty eyes, with AC depth above $3.50 \mathrm{~mm}$. All twenty eyes were implanted with ICL. In both types; the surgery was safe, effective, predictable and had a low complication rate. It also provided rapid visual rehabilitation and long term stability. Follow up of these eyes has been done for three months postoperative. We found that, The BCVA improved one to two lines from the preoperative values in both types and the refractive results of both type were stable. As for the endothelial cell count; the prcentage changes in $\mathrm{CD}$ is more with group $\mathrm{I}$ (AC depth< $3.50 \mathrm{~mm}$ ). Howerver No significant differences were found regarding the $\mathrm{CD}$ change, other parameters of corneal endothelium between group I and II. Both groups caused neither retinal detachment, nor pupillary block. Hence, ACD effect on both groups were equal as regards the effect on corneal endothelium and postoperative AC inflammation, predictability, efficacy and the effect on IOP.
\end{abstract}

Keywords: Implantable collamer lens, Cell density, Coefficient of variation, Anterior chamber depth.

\section{Introduction}

The corneal endothelium is the inner most layer of the cornea, 4 to $6 \mu \mathrm{m}$ thick [1], which is important for maintenance of normal corneal hydration, thickness and transparency by the function of its metabolic pump. The human corneal endothelium has little or no ability to divide after birth [1]. These cells are hexagonal in shape and approximately $20 \mu \mathrm{m}$ wide[1].

Adjacent cells share extensive lateral interdigitations, and also have gap and tight junctions along their lateral borders. The lateral membranes contain $\mathrm{Na}+/ \mathrm{K}+-\mathrm{ATPase}$ pump which is responsible for normal corneal hydration [2].

At birth normal cell density ranges from 35004000 cells $/ \mathrm{mm}^{2}$, adult cornea has densities of 1400- 2500 cells $/ \mathrm{mm}^{2}$ [1], normally the central ECD decreases by $0.3 \%$ per year [2].

Specular microscope is an useful non contact investigational technique that photographs the human corneal endothelium [3]. This instrument useful in detection of endothelial damage or disease under very high magnification which may not be seen by slit lamp examination, 100 times greater than slit lamp biomicroscopy [4].

The anterior chamber is the area between the cornea anteriorly and the iris and lens posteriorly. The diameter of anterior chamber varies from $11.3 \mathrm{~mm}$ to $12.4 \mathrm{~mm}$. Anterior chamber depth is variable (2.9-3.8 $\mathrm{mm})$, being larger in myopic eyes and shallower in hyperopes. Due to the increased axial width of the lens, it decreases with age $(0.1 \mathrm{~mm}$ per decade). The depth of anterior chamber is greatest axially, and becomes progressively shallower peripherally [5].

Historically, the implantable collamer lens (ICL) has been used for the treatment of high myopia due to the decreased efficacy and safety of laser in situ keratomileusis (LASIK) and photorefractive keratectomy (PRK) in these patients. Several clinical studied have demonstrated the superiority of ICL implantation for the correction of moderate to high myopia. Otherwise, the most commonly documented safety concerns of ICL implantation are mild endothelial cell loss, increased IOP, pupillary block, and cataract formation as a result of inadequate postoperative ICL vault $[6,7]$.

In recent studies, rotating Scheimpflug imaging (Pentacam), anterior segment OCT, and UBM have been used to measure the phakic IOL vault and have all provided accurate and reproducible measures of anterior segment biometry. In our present study, we used the rotating Scheimpflug imaging system (Pentacam) that has proved to be a repeatable measurement to evaluate the vault [8].

\section{Methodology}

Five electronic databases were used to find relevant studies published in English.

\subsection{Search Strategy}

By September 2018, we conducted a search on five electronic databases, including PubMed, Scopus, Institute of Science Index (ISI), Google Scholar and Cochrane. We used the following 
search terms to retrieve the articles that may relate to our topic: (anterior chamber depth, corneal endothelial cells).

\subsection{Statistical methods}

The collected data was revised, coded, tabulated and introduced to a PC using Statistical package for Social Science from International Business Machines corporation (IBM Corp. Released 2017. IBM SPSS Statistics for Windows, Version 25.0. Armonk, NY: IBM Corp.). Data were presented and suitable analysis was done according to the type of data obtained for each parameter. Analytical statistics: Student $\mathrm{T}$ Test was used to assess the statistical significance of the difference between two study group means.

Repeated measure ANOVA was used to assess changes in parameters over time. All tests were 2-sided and a P-value $<0.05$ was considered statistically significant, P-value> 0.05 was considered not statistically significant.

\subsection{Inclusions criteria}

Patients aged from $20 \mathrm{y}$ to $35 \mathrm{y}$, Patients with endothelial cell count more than 2000 cells/ mm2. Patients with high emmetropia not suitable for laser refractive surgery. Patients with anterior chamber depth ranging from $2.85 \mathrm{~mm}$ to $4.0 \mathrm{~mm}$ measured from corneal endothelium to the anterior lens capsule via the Oculus Pentacam HR. A pupil (under mesopic light conditions) smaller than $6.0 \mathrm{~mm}$. Horizontal White to white (WTW) distance is more than $11 \mathrm{~mm}$ in cases of ICL implantation. Skilled surgeon who already doing the technique involved in the surgery for more than 250 cases solely befor the study.

\subsection{Exclusion criteria}

Patients with anterior chamber depth below $2.85 \mathrm{~mm}$, abnormal pupil, fixed pupil or pupil in mesopic light greater than $6.5 \mathrm{~mm}$. White to white distance less than $11 \mathrm{~mm}$.

Previous ocular trauma or previous eye surgery.

Pre-existing diseases of the vitreous, macula, or optic nerve that can affect visual outcome. Patients with uveitis and anterior segment pathology.

\section{Results}

The current study was conducted on 20 eyes of 10 patients, $30 \%$ males and $70 \%$ female. Mean age \pm SD was 23.6 \pm 3.4 years, 10 left and 10 right eyes. Each group included 5 right and 5 left eyes.

Mean baseline UCVA Table (1) in all studied cases was 0.04 in all studied cases. No significant differences were found between group I and group II regarding UCVA preoperatively, 1 week, 1 month and 3 months postoperatively (p1>0.05 for each). While there were significant increase in UCVA across time points in all patients, as well as in group I and group II Fig (1).

Mean baseline SE in all studied cases was 10.35 in all studied cases. No significant differences were found between group I and group II regarding SE preoperatively, 1 week, 1 month and 3 months postoperatively (p1>0.05 for each).

While there were significant trend of decrease in SE across time points in all patients, as well as in group I and group II (p2<0.001 for each).

Mean baseline BCVA in all studied cases was 0.68 in all studied cases. No significant differences were found between group I and group II regarding BCVA preoperatively, 1 week, 1 month and 3 months postoperatively ( $1>0.05$ for each). While there were significant increase in BCVA across time points in all patients, as well as in group I and group II (p2<0.001 for each).

Mean baseline IOP in all studied cases was 13.8 in all studied cases. No significant differences were found between group I and group II regarding IOP preoperatively, 1 week, 1 month and 3 months postoperatively ( $1>0.05$ for each). In addition, no significant differences were found in IOP across time points in all patients, as well as in group I and group II (p2>0.05 for each).

Mean baseline CCT in all studied cases was 488.1 in all studied cases. Group II showed significantly higher CCT when compared to group I preoperatively, 1 week, 1 month and 3 months postoperatively $\quad(\mathrm{p}=0.01,0.004,0.005,0.003$ respectively). While there were no significant differences regarding CCT across time points in all patients, as well as in group I and group II (p2>0.05 for each).

Mean baseline $\mathrm{CV}$ in all studied cases was 35.5 in all studied cases. No significant differences were found regarding $\mathrm{CV}$ between group I and II at each time point (p1>0.05 for each). Moreover, no significant differences were found across time in CV in all cases, group I and group II ( $p>0.05$ for each).

Mean baseline Hex in all studied cases was 52 in all studied cases. No significant differences were found regarding Hex between group I and II at each time point (p1>0.05 for each). Moreover, no significant differences were found across time in Hex in all cases, group I and group II ( $>0.05$ for each).

Mean baseline Avg in all studied cases was 325.6 in all studied cases. No significant differences were found regarding Avg between group I and II at each time point (p1>0.05 for each). Moreover, no significant differences were found across time in Avg in all cases, group I and group II ( $p>0.05$ for each).

Mean baseline $\mathrm{CD}$ in all studied patients was 3082.5 Table (2). No significant differences were found regarding $\mathrm{CD}$ between group $\mathrm{I}$ and II at 
each time point (p1>0.05 for each). Moreover, no significant differences were found across time in $\mathrm{CD}$ in all cases, group I and group II Fig (2).

Percentage changes in CD Table (3) in relation to baseline levels was calculated. CD decreased after 1 week by $0.3 \%$, after 1 month by $1.2 \%$ and was settled to the 3rd month. Regarding group I, CD decreased after 1 week by $1.3 \%$, after 1 month by $2.9 \%$ and after 3 months by
3\%. Regarding group II, CD increased after 1 week by $0.7 \%$, while decreased after 1 month by $4 \%$ and was settled to the 3rd month. No significant differences were found regarding $\mathrm{CD}$ change between group I and II at each time point ( $\mathrm{p} 1>0.05$ for each). Moreover, no significant differences were found across time in CD change in all cases, group I and group II Fig. (3).

Table (1) Comparison of UCVA between studied groups across all studied time points.

\begin{tabular}{|c|c|c|c|c|c|c|c|c|}
\hline & & \multicolumn{2}{|c|}{$\begin{array}{c}\text { All patients } \\
\mathbf{N}=20\end{array}$} & \multicolumn{2}{|c|}{$\begin{array}{c}\text { Group I } \\
\mathbf{N}=10\end{array}$} & \multicolumn{2}{|c|}{$\begin{array}{c}\text { Group II } \\
\mathrm{N}=10\end{array}$} & \multirow[t]{2}{*}{$\mathbf{P}^{1}$} \\
\hline & & mean & SD & mean & SD & mean & SD & \\
\hline \multirow[t]{5}{*}{ UCVA } & preoperative & 0.04 & 0.01 & 0.05 & 0.01 & 0.03 & 0.01 & 0.220 \\
\hline & 1 week Post-Operative & 0.72 & 0.17 & 0.73 & 0.21 & 0.70 & 0.14 & 0.708 \\
\hline & 1 month Post-Operative & 0.77 & 0.20 & 0.76 & 0.24 & 0.77 & 0.17 & 0.915 \\
\hline & 3 months Post Operative & 0.78 & 0.19 & 0.80 & 0.21 & 0.76 & 0.18 & 0.647 \\
\hline & $\mathbf{P}^{2}$ & \multicolumn{2}{|c|}{$<0.001$} & \multicolumn{2}{|c|}{$<0.001$} & \multicolumn{2}{|c|}{$<0.001$} & \\
\hline
\end{tabular}

$\mathrm{P} 1$, comparison between group I and II using $\mathrm{t}$ test; $\mathrm{p} 2$, comparison across time points using repeated measure ANOVA; SD, standard deviation.

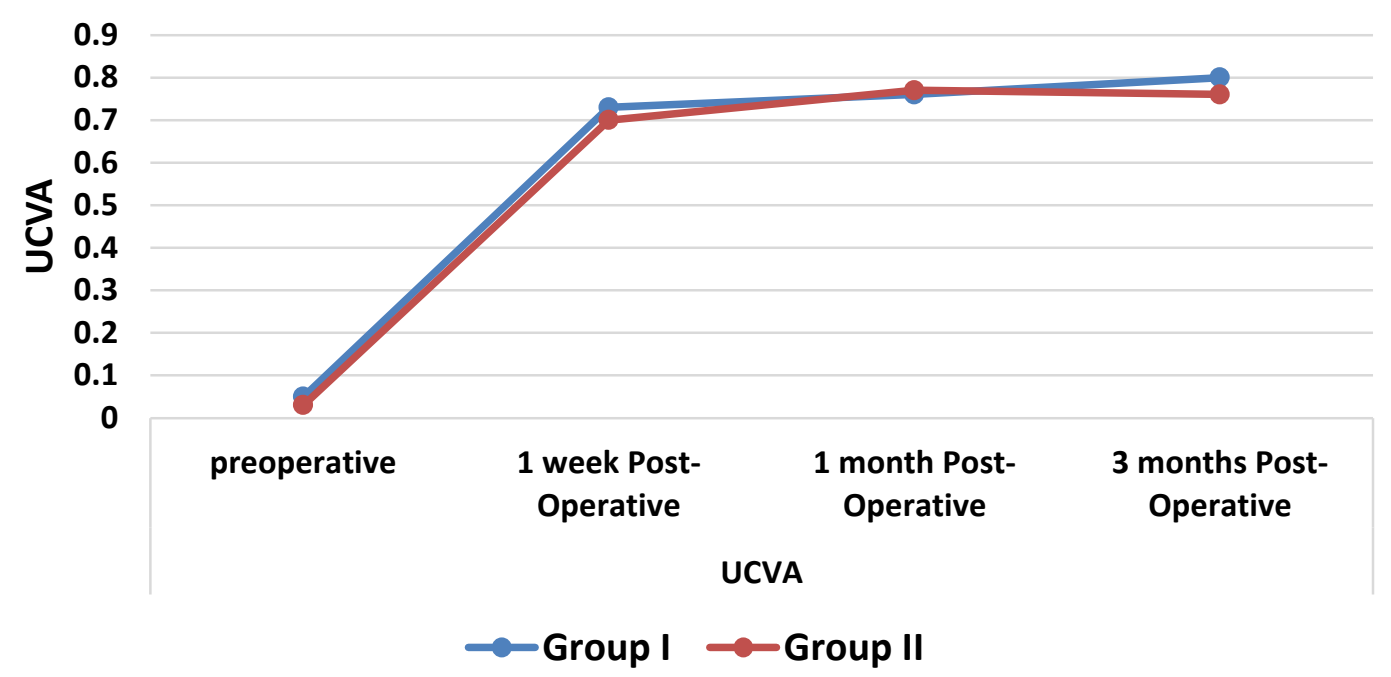

Fig (1) UCVA among studied groups across all studied time points.

Table (2) Comparison of $\mathrm{CD}$ between studied groups across all studied time points.

\begin{tabular}{llccccccc}
\hline & & \multicolumn{2}{c}{ All patients } & \multicolumn{2}{c}{ Group I } & \multicolumn{2}{c}{ Group II } & \multirow{2}{*}{$\mathbf{P}^{\mathbf{1}}$} \\
& & \multicolumn{2}{c}{$\mathbf{N = 2 0}$} & \multicolumn{2}{c}{$\mathbf{N = 1 0}$} & \multicolumn{2}{c}{$\mathbf{N = 1 0}$} & \\
\cline { 2 - 7 } & mean & SD & mean & SD & mean & SD & \\
\hline CD & preoperative & 3082.5 & 193.3 & 3103.2 & 165.7 & 3061.7 & 224.6 & 0.644 \\
& 1 week Post Operative & 3019.6 & 282.6 & 2972.2 & 356.7 & 3067.0 & 190.7 & 0.468 \\
& 1 month Post-Operative & 2943.7 & 307.3 & 2862.2 & 391.4 & 3025.2 & 177.1 & 0.246 \\
& 3 months Post-Operative & 2970.2 & 274.0 & 2887.1 & 334.3 & 3053.3 & 177.1 & 0.182 \\
\multicolumn{2}{c}{$\mathbf{P}^{2}$} & \multicolumn{2}{c}{0.067} & \multicolumn{2}{c}{0.082} & \multicolumn{2}{c}{0.738} & \\
\hline
\end{tabular}

$\mathrm{P} 1$, comparison between group $\mathrm{I}$ and II using $\mathrm{t}$ test; $\mathrm{p} 2$, comparison across time points using repeated measure ANOVA; SD, standard deviation. 


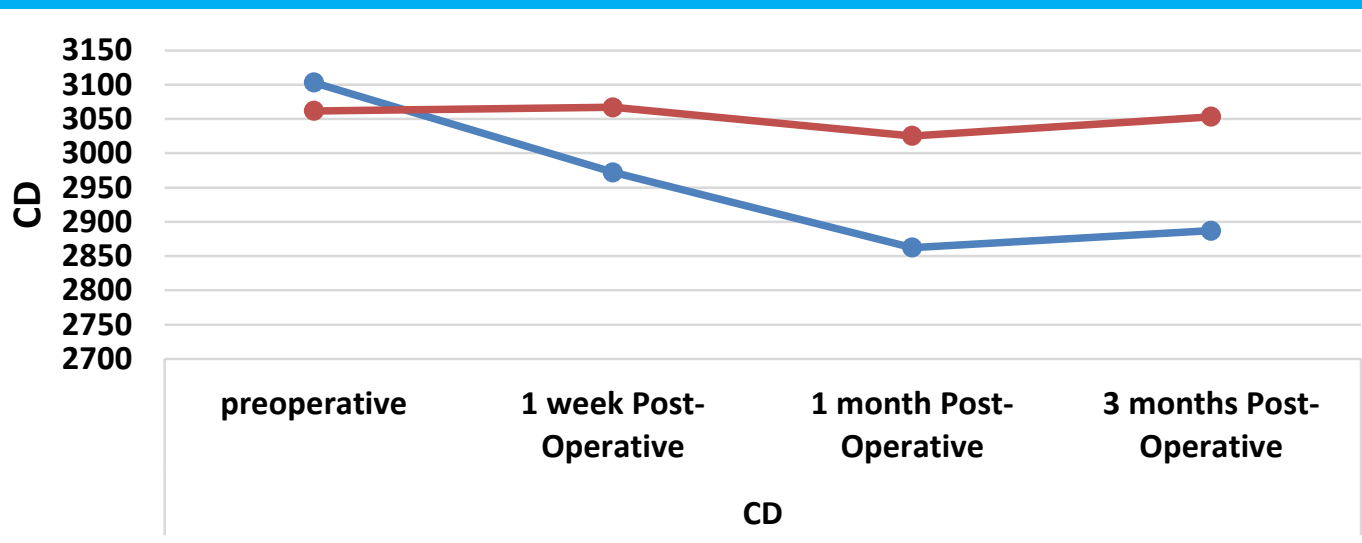

Group I Group II

Fig (2) CD among studied groups across all studied time points.

Table (3) Comparison of CD percentage change between studied groups across all studied time points.

\begin{tabular}{cccccc}
\hline & \multirow{2}{*}{ Change from baseline } & All patients & Group I & Group II & N=10 \\
N=20 & N=10 & $-1.3 \%$ & $0.7 \%$ & 0.705 \\
CD & 1 week Post-Operative & $-0.3 \%$ & $-2.9 \%$ & $-0.4 \%$ & 0.174 \\
& 1 month Post-Operative & $-1.2 \%$ & $-3 \%$ & $-0.4 \%$ & 0.131 \\
& 3 months Post-Operative & $-1.2 \%$ & 0.273 & 0.905 & \\
\hline
\end{tabular}

$\mathrm{P} 1$, comparison between group I and II using $\mathrm{t}$ test; $\mathrm{p} 2$, comparison across time points using repeated measure ANOVA; SD, standard deviation.

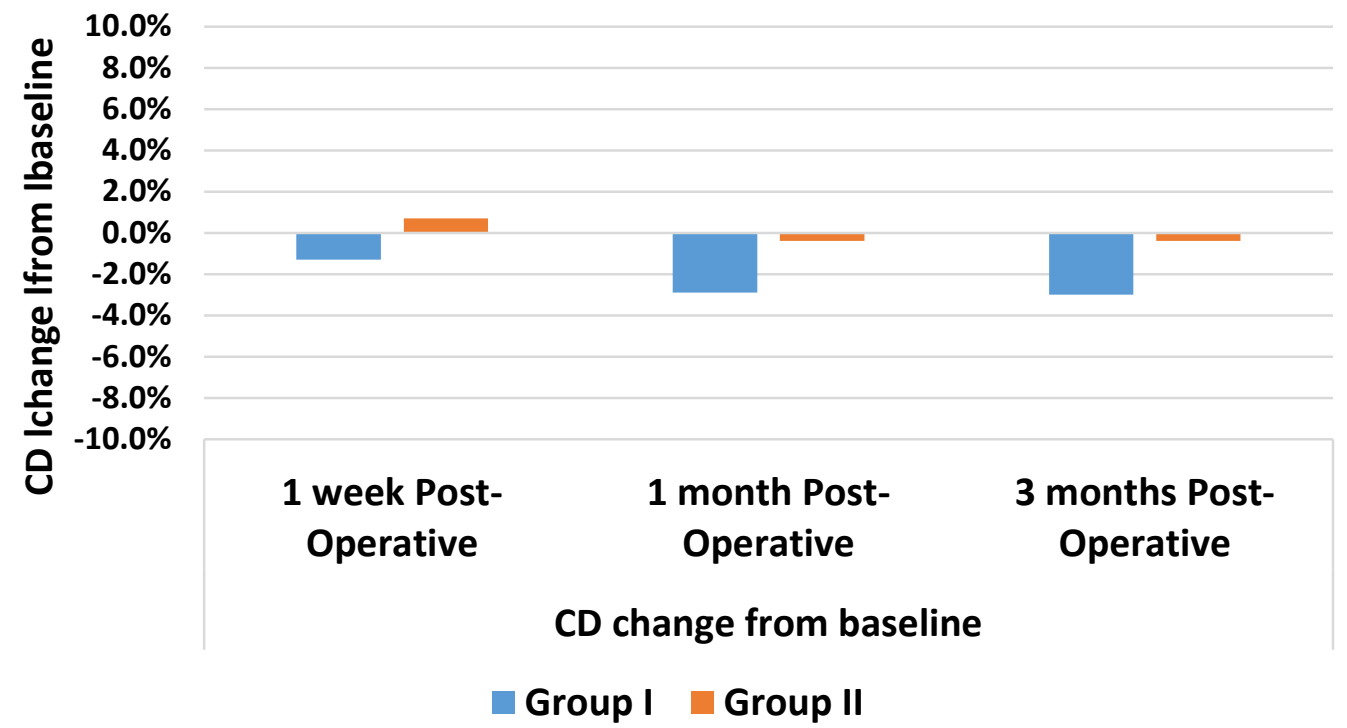

Fig (3) CD change from baseline among studied groups across all studied time points.

\section{Discussion}

Phakic IOLs demonstrate reversibility, high optical quality, and potential gain in visual acuity in high emmetropia due to retinal magnification; also they are not limited by corneal thickness or topography [9].
The drawbacks of phakic IOLs are rare and related to pIOL position and type. The main complications of PC pIOLs are: anterior subcapsular cataract formation, chafing of the posterior iris and ciliary processes with pigmentary dispersion syndrome, damage to the zonules with dislocation of the IOL in the 
vitreous, pupillary block glaucoma and malignant glaucoma, chronic uveal inflammation, and macular edema [10)]. No causative relationship between pIOL implantation (of any pIOL type) and retinal detachment has been established [9].

For pIOLs to be clinically acceptable, they must achieve a high standard of efficacy, predictability, stability and safety. UCVA is the main index used to assess the effectiveness of a refractive procedure [11]. The results we got in our study concerning effectiveness, predictability, stability and improvement in visual acuity were similar to many previous studies of phakic IOLs.

After 3 months follow up, 19 eyes (95\%) in both groups had an UCVA of 6/12 (0.5) or better. Regarding final refraction; 7 eyes $(35 \%)$ were emmetrope with UCVA of 6/6 (0.9-1.0), 13 eyes $(65 \%)$ were within $\pm 0.5 \mathrm{D}, 7$ eyes $(35 \%)$ were within $\pm 1.75 \mathrm{D}$. The refractive results were stable and the BCVA improved one to two lines from the preoperative values.

Similarly; in a United States FDA study, the ITM study group showed that the ICL pIOL had good safety, efficacy and functional results with a low complication rate [12]. Regarding pIOL rotation or dislocation; no cases of ICL dislocation or rotation had occurred in our study due to accurate WTW measurement which was done using the Scheimpflug photography and by using a caliber while the patient was laying supine [13].

Yet, Trindade and Pereira, 2000; reported exchanging an ICL because of oversized length. Malpositioning with a very large vault and under correction occurred because the ICL was too long. The ICL was exchanged for a smaller ICL with higher power [14]. In a study with a 12month follow-up, UBM showed ICL rotation in $11 \%$ of eyes. Although there was no decentration of the optic, the authors suggest that the diameter of the ICL was too small [15].

Although; one of the most common expected complications after pIOLs is cataract formation, yet; we did not report any case of cataract formation after ICL over the follow up period. This could be attributed to proper ICL vault, proper measuring of white to white distance which was measured using using the Scheimpflug photography.

The same study by Zaldivar et al., 1998; concluded that non of 124 eyes developed lens opacities due to ICL implantation. Nevertheless, one eye developed peripheral lens opacification at the position where $\mathrm{Nd}$ :YAG iridotomy which was performed preoperatively [16].

On the Contrary; Sanders et al, 2003, stated that $0.6 \%$ developed significant lens opacity in the ICL's FDA trial. On the other hand, found that the incidence of ICL-induced anterior subcapsular cataract was $27 \%$. Also, these anterior subcapsular opacities were described by Uusitalo et al., 2002; who clarified that The reason why these cataracts develop is not well established, although it is widely believed that it depends mainly on the space (vault) between the IOL and the natural lens. When this space is too narrow or even nonexistent (the IOL touching the natural lens) the aqueous humor cannot flow freely around the lens, causing changes in metabolism responsible for the opacities. While Ghanem and Azar, 2008; stated that metabolic disturbances induced by the implant might also be partially responsible for cataract formation. However, a longer follow up is needed as it may detect more cases of cataract [17-19].

No cases of RD occurred in our study, with both groups. Mostly this was due to thorough preoperative and postoperative fundoscopic investigation. On the contrary; Panozzo and Parolini, 2001; recorded four cases of RD after ICL, Zaldivar et al., 1998; reported a single case of RD after implantation of a PC pIOL in 124 eyes [16, 20].

The present study revealed the ICL vault (distance from the anterior lens surface and the center of ICL optic from the posterior surface) was within the ideal range [from $1 / 2$ CCT to $1 \frac{1 / 2}{2}$ CCT $(250 \mu \mathrm{m}$ to $750 \mu \mathrm{m})]$ in all cases of both groups. High vault is considered when the vault is more than $750 \mu \mathrm{m}$ while a low vault if less than $250 \mu \mathrm{m}$.

Similar to our study; El Danasoury, 2010 found that the ICL sits further away from the corneal endothelium than AC pIOLs and he concluded that ACD of $2.7 \mathrm{~mm}$ from the endothelium to the anterior surface of the crystalline lens is estimated as the lower limit for safe ICL implantation [21]. Moreover, anteroposterior movement of the ICL during iris contraction or accommodation led to intermittent central contact [15].

Another rare complication is implantation of a pIOL with incorrect power. Due to the aim of the surgery is to correct ametropia as precisely as possible, this complication should not occur with current formulas [9].

The major statistically significant finding in our study was the decrease in the endothelial cell count (ECC) in both groups. The decrease in the ECC in the ICL was only attributed to direct trauma to the endothelium during surgery.

Percentage changes in $\mathrm{CD}$ in relation to baseline levels was calculated. CD decreased after 1 week by $0.3 \%$, after 1 month by $1.2 \%$ and was settled to the 3rd month. Regarding group I, CD decreased after 1 week by $1.3 \%$, after 1 month by $2.9 \%$ and after 3 months by $3 \%$. Regarding group II, CD increased after 1 week by $0.7 \%$, while decreased after 1 month by $4 \%$ and was settled to the 3rd month.

In the present study, it was hypothesized that ACD could be a risk factor for increasing 
endothelial cell loss after ICL. As such, the percentage of corneal endothelial cell loss was higher in the group I than in group II. However $\underline{\text { no significant differences were found regarding }}$ $\underline{\mathrm{CD}}$ change between group I and II at each time point (p1>0.05 for each). Moreover, no significant differences were found across time in CD change in all cases, group I and group II $(p>0.05$ for each).

Natural loss of corneal endothelial cells is about $0.6 \%$ per year [22)]. In contrast to our findings; a more endothelial cell loss of $5.2 \%$ $5.5 \%$ was documented after 12 months [17], an endothelial cell loss of $8.4-9.7 \%$, three years postoperative [12]. Researchers therefore considered surgery to be the cause of the early corneal endothelial cell loss for pIOL. This is also affected by the learning curve of the surgeon [23].

Factors leading to corneal endothelial cell loss after pIOL implantation, were studied by many authors who reported a yearly corneal endothelial cell loss of $1.0 \%$ for a mean minimum distance of $1.43 \mathrm{~mm}$ between the edge of the pIOL and the corneal endothelium; the loss was $1.7 \%$ for a mean minimum distance of $1.20 \mathrm{~mm}$ and $0.2 \%$ for a mean minimum distance of $1.66 \mathrm{~mm}$. They also expected that a critical corneal endothelial cell level of 1500 cells $/ \mathrm{mm}^{2}$ would be reached 18 years after Artisan/Artiflex implantation [24].

Our study had some limitations. The enrolled patients were only followed for 3 months. Thus, a long-term study is needed. In addition, our study could not have a blinded design, so other studies with this design are needed. Furthermore, more patients should be enrolled in future studies.

A significant strength of the study is its design, as to the best of our knowledge; this is the first controlled and stratified study to describe the relationship between ACD and corneal endothelial cell loss after implantable collamer lens (ICL) surgery.

We demonstrated that a shallow ACD is related to endothelial cell loss in ICL surgery. Thus, refractive surgeons should pay particular attention to patients with shallow ACDs during ICL surgery.

UIS is synonymous with ulno-carpal impaction syndrome and ulno-carpal abutment, but is a distinct entity from ulnar styloid impaction and ulnar impingement syndrome. Ulnar styloid impaction is caused by impaction between the ulnar styloid and the proximal triquetrum; ulnar impingement syndrome is defined by a shortened ulna impinging on the distal radius [5]. UIS may lead to degen-erative lesions of the triquetrum, lunate, ulnar head cartilage, or of the TFCC. Concomitantly the triquetro-lunate ligaments may be disrupted. Associated factors of ulno-carpal joint space narrowing may include previous fracture (e.g. of the distal radius), premature physeal arrest of the distal radius, or congenital ulna positive variance.

\section{Conclusion}

Our study revealed that phakic PC IOLs implantation (ICL) in moderate to high myopes had excellent results including; stability of refraction for high emmetropia, reversibility, high optical quality, potential gain in visual acuity, preservation of corneal architecture, asphericity and accommodation. Moreover, correction is not limited by corneal thickness or topography.

However, selection depends on some criteria including; preoperative refraction, anterior Chamber Depth (ACD) and pupil size.

Regarding the preoperative refraction; ICL corrects upto -20.00D spherical error (spectacle plane).

Regarding ACD; a sufficient Anterior Chamber Depth is necessary. ACD less than $2.8 \mathrm{~mm}$ measured from the endothelium is considered a limitation for ICL. So we recommend ICL implantation in eyes with ACD $2.8 \mathrm{~mm}$ or more.

We also concluded that there are some precautions that should be made to avoid unnecessary complications of pIOLs. Among these precautions is; Preoperative Endothelial Cell Count (ECC) measurement, using specular microscopy, is obligatory. Patients with endothelial damage or ECC below 2000 cell $/ \mathrm{mm}^{2}$ should not receive a pIOL (ICL).

In addition; a thorough preoperative and postoperative fundoscopic examination is required to rule out retinal changes or vitreoretinal pathologies and to perform prophylactic laser photocoagulation, if required.

Also, corneal endothelial damage is also a possible complication. Our study proved that the prcentage changes in $\mathrm{CD}$ is more with group I (AC depth $<3.50 \mathrm{~mm}$ ). Howerver No significant differences were found regarding the $\mathrm{CD}$ change, other parameters of corneal endothelium between group I and II.

Finally, this study proved that regarding the postoperative visual acuity, glare, halos, IOP, and complications both groups had similar results.

\section{References}

[1] H.E.Kaufman, B.A.Barron, and M.B.McDonald, "Structure and Function of the Cornea." The Cornea. Second ed. Boston, MA: ButterworthHeinemann, PP.19-22, 1999.

[2] M.Yanoff, and J.S.Duker. "Cornea and Corneal Surface Diseases." Ophthalmology. Fourth ed. Edinburgh: Elsevier/Saunders, PP.164-67, 2014

[3] J. Kanski, B.Bowling B, K.Nischal, and Andrew Pearson. "Cornea." Clinical Ophthalmology: A Systematic Approach. Seventh ed. Edinburgh: Elsevier/Saunders, PP.168,172, 2011. 
[4] N.R.Adames, and N.A.Afshari. "The Changing Fate of the Corneal Endothelium in Cataract Surgery." Current Opinion in Ophthalmology, Vol.23.1, PP.3-6, 2012.

[5] J.G.Lawrenson, Production and drainage of aqueous humour - Glaucoma identification and co management, Edgar DF and Rudnicka AR (Ed.), Butterworth-Heinemann, an imprint of Elsevier Inc, PP.17-26, 2007.

[6] P.Fernandes, J.M.Gonzalez-Meijome, D. Madrid-Costa , T.Ferrer-Blasco, J.Jorge, R.Montes-Mico, "Implantable collamer posterior chamber intraocular lenses: a review of potential complications." $J$ Refract Surg; Vol.27(10), PP.765-776, 2011.

[7] T.Kohnen, D.Kook, M.Morral, JLGuell, "Phakic intraocular lenses: part 2: results and complications." J Cataract Refract Surg ,Vol.36(12), PP.2168-2194,2010.

[8] K.Kamiya et al., "Comparison of vault after implantation of posterior chamber phakic intraocular lens with and without a central hole." J Cataract Refract Surg. , Vol.41(1), PP.67-72, 2015.

[9] T.Kohnen, D.Kook, M.Morral, J.L.Guell, "Phakic intraocular lenses: part 2: results and complications." J Cataract Refract Surg ,Vol.36(12), PP.2168-2194, 2010.

[10] L.Werner, D.J.Apple, S.K.Pandey, R.H.Trivedi, A.M.Izak and T.A.Macky, Phakic posterior chamber intraocular lenses. Int Ophthalmol Clin, Vol.41, PP.153-174, 2001.

[11] J.J.Pérez-Santonja, J.L.Bueno, J.Meza, B.García-Sandoval, J.M.Serrano and M.A.Zato: Ischemic optic neuropathy after intraocular lens implantation to correct high myopia in a phakic patient. J Cataract Refract Surg, Vol.19, PP.651-4, 1993.

[12] ITM.ICL in Treatment of Myopia Study Group.: United States Food and Drug Administration clinical trial of the Implantable Collamer Lens (ICL) for moderate to high myopia; three-year follow-up. Ophthalmology.; Vol.111:PP.1683-1692, 2004.

[13] J.L.Menezo, C.Peris-Martínez, A.Cisneros and R.Martínez-Costa, Posterior chamber phakic intraocular lenses to correct high myopia: a comparative study between Staar and Adatomed models. J Refract Surg, Vol.17, PP.32-42, 2001.

[14] F.Trindade and F.Pereira, Exchange of a posterior chamber phakic intraocular lens in a highly myopic eye. J Cataract Refract Surg, Vol.26, PP.773-776, 2000.

[15] J.García-Feijoó, I.Jiménez-Alfaro, R.CuiñaSardiña, J.M.Benítez del Castillo, C.MéndezHernández and J.García-Sanchez, Ultrasound biomicroscopy examination of posterior chamber phakic intraocular lens position. Ophthalmology 200, Vol.110, PP.163-172,2003.

[16] R.Zaldivar, J.M.Davidorf and S.Oscherow, Posterior chamber phakic intraocular lens for myopia of -8 to -19 diopters. J Refract Surg Vol.14, PP.294-305, 1998.

[17] D.R.Sanders, J.A.Vukich, K.Doney and M.Gaston, Implantable Contact Lens in Treatment of Myopia Study Group. US Food and Drug Administration clinical trial of the implantable contact lens for moderate to high myopia. Ophthalmology, Vol.110(2), PP.255266,2003.

[18] R.J.Uusitalo, E.Aine, N.H.Sen and L.Laatikainen, Implantable contact lens for high myopia. J Cataract Refract Surg. ,Vol.28, PP.29-36, 2002.

[19] R.C.Ghanem,and D.T.Azar, Yanoff and Duker, Ophthalmology, 3rd ed edition, Chapter 3.10 Phakic Intraocular Lenses, 2008.

[20] G. Panozzo and B.Parolini, Relationships between vitreoretinal and refractive surgery. Ophthalmology,Vol.108, PP.1668-1669,2001.

[21] M.A.El Danasoury, Special Indications for the Use of Phakic IOLs. Posterior chamber phakic IOLs can be used in patients with stable keratoconus and other indications beyond myopia and myopic astigmatism. CATARACT \& REFRACTIVE SURGERY TODAY EUROPE. Feb, PP.57-62, 2010.

[22] W.M.Bourne, L.R.Nelson and D.O.Hodge: Central corneal endothelial cell changes over a ten-year period. Invest. Ophthalmol. Vis. Sci, Vol.38, PP.779-782,1997.

[23] I.Dejaco-Ruhswurm, U.Scholz, S.Pieh, G.Hanselmayer, B.Lackner, C.Italon, M.Ploner and C.Skorpik: Long-term endothelial changes in phakic eyes with posterior chamber intraocular lenses. J Cataract Refract Surg, Vol.28, PP.1589-1593, 2002.

[24] M.Doors , J.De Brabander, F.Hendrikse and CAB.Webers and R.M.Nuijts, Influence of anterior chamber morphometrics on endothelial cell changes after phakic intraocular lens implantation. J Cataract Refract Surg, Vol.34, PP.2110-2118, 2008. 